\title{
Mechanisms of enhancement of light emission in nanostructures of II-VI compounds doped with manganese
}

\author{
M. Godlewski ${ }^{1,2}$, S. Yatsunenko ${ }^{1}$, and V.Yu. Ivanov ${ }^{1}$ \\ ${ }^{1}$ Institute of Physics Polish Academy of Sciences, Al. Lotnikow 32/46, 02-668 Warsaw, Poland \\ ${ }^{2}$ Department of Mathematic and Natural Sciences College \\ Cardinal S. Wyszynski University, Warsaw, Poland \\ E-mail: godlew@ifpan.edu.pl \\ K. Drozdowicz-Tomsia and E.M. Goldys \\ Division of Information and Communication Sciences, Macquarie University, Sydney, Australia \\ M.R. Phillips \\ Microstructural Analysis Unit, UTS, Sydney, Australia

\section{P.J. Klar and W. Heimbrodt} \\ Department of Physics and Materials Sciences Center, \\ Philipps-University of Marburg, Renthof 5, 35032 Germany
}

Received September 7, 2006

\begin{abstract}
Intra-shell transitions of transition metal and rare earth ions are parity forbidden processes. For $\mathrm{Mn}^{2+}$ ions this is also a spin forbidden process, i.e., light emission should be inefficient. Surprisingly, it was reported that in nanostructures of $\mathrm{ZnMnS}$ the ${ }^{4} T_{1}$ to ${ }^{6} A_{1}$ intra-shell transition of $\mathrm{Mn}^{2+}$ results in a bright photoluminescence characterized by a short PL decay time. The model of a quantum confined atom was introduced to explain the observed experimental results. It was later claimed that this model is incorrect. Based on the results of our photoluminescence, photoluminescence kinetics, time-resolved photoluminescence, electron spin resonance and optically detected magnetic resonance investigations we confirm photoluminescence enhancement and decrease of photoluminescence lifetime and relate these effects to spin dependent magnetic interactions between localized spins of $\mathrm{Mn}^{2+}$ ions and spins/magnetic moments of free carriers. This mechanism is active in both bulk and in low-dimensional structures, but is significantly enhanced in nanostructure samples.
\end{abstract}

PACS: 81.07.Wx Nanopowders;

78.55.Et II-VI semiconductors;

78.47.+p Time-resolved optical spectroscopies and other ultrafast optical measurements in condensed matter;

76.70.Hb Optically detected magnetic resonance.

Keywords: nanocrystals, photoluminescence, magnetic resonance.

\section{Introduction}

Recently a new application of small (below $10 \mathrm{~nm}$ ) nanocrystals was proposed. They can be used for fluo- rescence labeling for monitoring biochemical reactions and for their quantitative analysis. Some of such biological applications are described in our recent review [1]. We stressed there that nanocrystals should be of 
nanometer size, since many object we plan to label are equally small. For example, cells typically range from several micrometers to a few tens of nanometers.

The fluorescent labels presently used have high quantum efficiency of the light emission, but their application is limited by relatively narrow excitation region, small separation between excitation and emission energies, broad emission, and sensitivity to photo-bleaching [1]. Most of these limitations can be avoided by the use of semiconductor-based nanocrystals. These nanocrystals, when of nanometer sizes, have properties dictated by quantum confinement effects. To get spectrally narrow emission nanocrystals can be doped with common emission activators, such as transition metal or rare earth (RE) ions. However, intra-shell $3 d-3 d$ or $4 f-4 f$ transitions suffer from parity selection rules. Moreover, in some cases these transitions are also spin forbidden, which further limits rate of radiative recombination. For example, the ${ }^{4} T_{1}$ to ${ }^{6} A_{1}$ intra-shell transition of $\mathrm{Mn}^{2+}$ ions is a parity and spin (transition between spin quartet and sextet) forbidden process. Thus, this transition is fairly inefficient in bulk samples, especially in sulfides and selenides, i.e., in wide-band-gap II-Mn-VI compounds with weak spin-orbit interactions.

One should relax both parity and spin selection rules to increase the recombination rate of the intra-shell PL. Both these rules are partly relaxed in II-VI compounds with a strong spin-orbit interaction. This is why shorter PL decay times were observed for the ${ }^{4} T_{1}$ to ${ }^{6} A_{1}$ intra-shell transition of $\mathrm{Mn}^{2+}$ ions in tellurides (CdTe and ZnTe) [2]. The PL decay time for the $\mathrm{Mn}^{2+}$ intra-shell transition shortens from milliseconds, observed in $\mathrm{ZnMnS}[3,4]$, to microseconds in CdMnTe and ZnMnTe [2].

Very different PL properties were reported for $\mathrm{Mn}$ and RE doped nanocrystals [5-9]. In a series of papers Bhargava and co-workers [5-9] reported distinctly different PL properties for doped nanocrystals of sizes below $10 \mathrm{~nm}$. In case of ZnMnS nanocrystals the observed PL decay time of the ${ }^{4} T_{1}$ to ${ }^{6} A_{1}$ transition was shorter by 5 orders in magnitude. PL decay times of 3.7 and 20.5 ns were observed instead of millisecond ones [5]. Such lifetime shortening was observed together with an increase in emission efficiency, i.e., the effect could not be related to an enhanced rate of nonradiative decay [5].

The quantum confined atom (QCA) model was introduced to explain the shortening of the PL decay for either $\mathrm{Mn}^{2+}$ or RE ions [5-9]. Actually the QCA model proposed in a series of papers varied slightly, including the one based on the electron spin resonansce (ESR) investigations [6]. In that study it was proposed that the shortening of the PL lifetime is due to axial fields near the surface of nanocrystals.
For small nanocrystals a significant part of the $\mathrm{Mn}^{2+}$ ions is in a near-surface region, i.e., they are located in electric fields of a lower symmetry. A crystal field of low-symmetry admixes states of different parity and spin multiplicity to states participating in the PL transitions. Thus, such admixture relaxes both parity and spin selection rules. This is why $\mathrm{Mn}^{2+}$ ions from surface regions should strongly influence the observed PL emission. However, such an explanation of the data was not repeated in further reports.

In the originally proposed QCA model $[5,8]$ quantum confinement leads in small nanocrystals to a hybridization of Mn impurity $d$ wave functions with the host $s$ - and $p$-like states. As the consequence, $\mathrm{Mn}^{2+}$ intra-shell PL is no longer a spin- and parity-forbidden process, and the recombination rate is enhanced. A similar idea was used to account for a fast and very intensive PL decay observed for $\mathrm{Eu}$ and $\mathrm{Tb}$ doped $\mathrm{Y}_{2} \mathrm{O}_{3}$. In this case it was assumed that quantum confinement influences excited states of RE ions ( $5 d$ and $6 s$ ) and thus increases the rate of $4 f-5 d$ and $4 f-6 s$ absorption, resulting in efficient energy pumping to RE ions [8].

This model and also the experimental results of Bhargava and co-workers were rejected by Bol and Meijerink [4]. Those authors claimed that the QCA model must be incorrect, since a fast component of the ${ }^{4} T_{1}$ to ${ }^{6} A_{1}$ PL decay is observed together with a «normal» one in the millisecond time range, which in the opinion of those authors was impossible to explain in the framework of the QCA model.

However, this may not be the correct reasoning if we consider the QCA model proposed in the Ref. 7. In that paper Bhargava and co-workers claimed that the high quantum efficiency of the PL transition is a consequence of strong interaction between excited states of $\mathrm{Mn}^{2+}$ ions and photo-induced free carriers. Such interaction is strongly enhanced in nanocrystals due to quantum confinement imposed on free carriers. Consequently, a faster PL decay is expected only if free carriers are present. After their recombination a «normal» slow decay of the ${ }^{4} T_{1}$ to ${ }^{6} A_{1}$ intra-shell PL may be observed. If this explanation is correct, we can explain contradiction between results of Bhargava et al. [5-9] and Bol and Meijerink [4]. This hypothesis is verified in the present study.

\section{Experimental setups}

We used several excitation sources for PL, PL kinetics and time-resolved PL investigations. The latter two experiments were performed using a YAG:Nd laser and its harmonics (second, third, and fourth $-\lambda_{2}=532 \mathrm{~nm}$, $\lambda_{3}=355 \mathrm{~nm}, \lambda_{4}=266 \mathrm{~nm}$ ). Pulse duration was about $20 \mathrm{~ns}$. We used a very low pulse repetition frequency $f=30 \mathrm{~Hz}$, which allowed us to follow PL kinetics in 
a very wide time range, from ns to ms. Pulsed YAG: Nd laser was coupled with OPO system (optical parametrical oscillator) with a tuning range from 400 to $700 \mathrm{~nm}$. We could thus tune excitation energy in the PL investigations. A LeCroy fast oscilloscope was used to monitor a pulse half-width and pulse duration. Samples were mounted in a liquid helium cryostat with a tunable temperature range from 300 to $4.2 \mathrm{~K}$ controlled with a LakeShore thermo-controller. For the PL detection we used an MDR-23 double monochromator and either a Hamamatsu CCD or Hamamatsu photomultiplier.

Electron spin resonance investigations were performed with a Bruker 300 spectrometer, working at $9.5 \mathrm{GHz}$ (X-band system). The samples were mounted in the $\mathrm{TE}_{012}$ cavity in a gas flow cryostat working in a wide temperature range from 2 to $300 \mathrm{~K}$.

Optically detected magnetic resonance (ODMR) experiments were performed with either the Q-band system operating at 35-36 GHz or with the $60 \mathrm{GHz}$ system. In the Q-band system we could on-line measure both ODMR and ESR signals. Samples were mounted in the Oxford Instruments superconducting magnet SpectroMag with the field up to $7 \mathrm{~T}$ and a tunable temperature range from 300 to $2 \mathrm{~K}$. All the ODMR investigations were performed at $2 \mathrm{~K}$ temperature.

\section{Results and discussion}

\subsection{Do we see quantum confinement effects?}

In the QCA model the authors postulated that a quantum confinement results in a strong carrier-induced hybridization effects, which should affect not only the PL decay time, but also, if the effect is so strong as was proposed, a recombination energy of the ${ }^{4} T_{1}$ to ${ }^{6} A_{1}$ intra-shell transition. We checked the latter possibility by comparing PL and time-resolved PL spectra in bulk samples and in nanocrystals of $\mathrm{ZnMnS}$. The relevant results are shown in Figs. 1 and 2. The $\mathrm{Mn}^{2+}$ intra-shell emission in bulk samples and in nanocrystals is observed at the same energy both under the $\mathrm{CW}$ and pulsed excitation. This means that energy of the ${ }^{4} T_{1}$ to ${ }^{6} A_{1}$ transition is not changed by confinement and proposed strong $s p-d$ hybridization.

\subsection{Recombination dynamics}

As described in the Introduction the contradicting models were presented by Bhargava et al. [5-9] and Bol and Meijerink [4]. To verify or reject these models we performed PL kinetics investigations for several wide-band-gap II-VI compounds doped with Mn. Samples of different dimensionality were studied bulk ZnMnS (1\% Mn), ZnMnSe (below 0.5\% Mn), CdMnTe $(1 \%, 10 \%$, and $30 \% \mathrm{Mn})$, quantum wells of

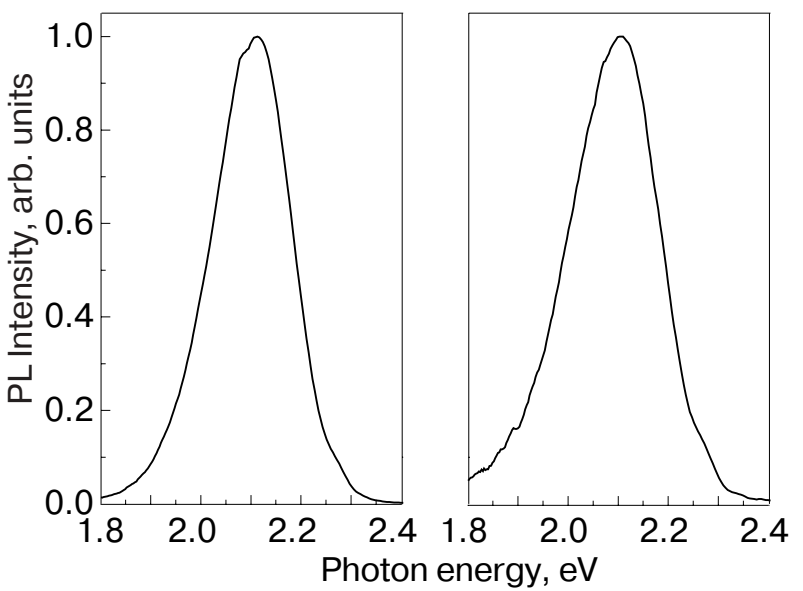

Fig. 1. Room temperature ${ }^{4} T_{1}$ to ${ }^{6} A_{1}$ PL spectra of bulk sample (left) and of nanocrystals (right) of $\mathrm{ZnMnS}$ with $1 \% \mathrm{Mn}$ fractions observed under the above band gap excitation.

CdMnTe/CdMgTe, quantum dots of CdMnTe and nanocrystals of $\mathrm{CdMnS}$ (from $1 \%$ to $30 \% \mathrm{Mn}$ ) and $\mathrm{ZnMnS}$ (from 1\% to 30\% Mn). Detailed description of the samples studied is given elsewhere [10-15] and thus is not repeated here. In this work we summarize the most important our observations and also present new data. The origin of the fast component of the PL decay is explained.

The most important result is that we observed the co-existence of fast and slow components of the PL decay in all the samples studied. The fast component of the decay is very enhanced in nanocrystals. This we show in Fig. 3, in which we compare fast components of the ${ }^{4} T_{1}$ to ${ }^{6} A_{1}$ PL decay for two $\mathrm{ZnMnS}$ samples with $1 \%$ Mn fraction, for a bulk sample and for

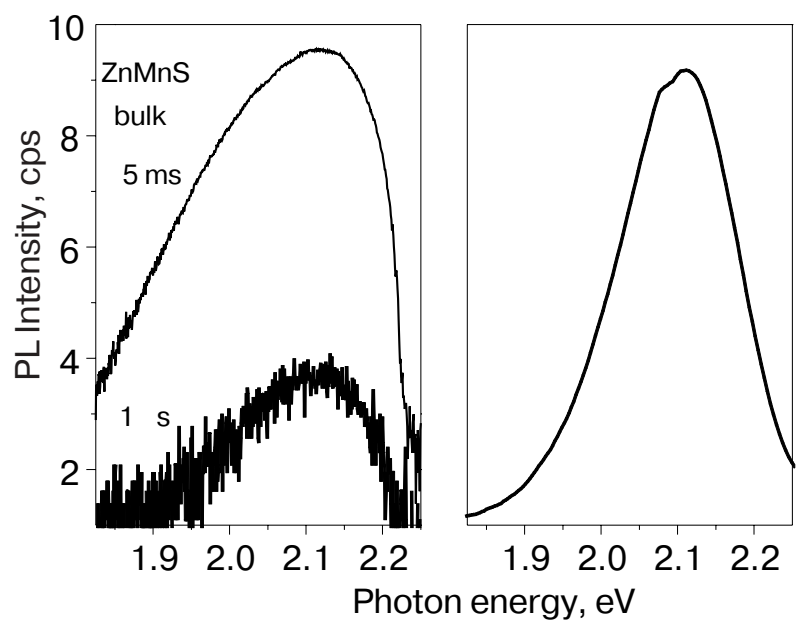

Fig. 2. Time-resolved PL spectra (left) measured for $\mathrm{ZnMnS}$ bulk sample with $1 \% \mathrm{Mn}$ fraction. The spectra collected through first $1 \mu$ s and 5 ms of the PL decay (after turning off the excitation) are compared. ${ }^{4} T_{1}$ to ${ }^{6} A_{1}$ $\mathrm{PL}$ emission (right) is shown for the comparison. 


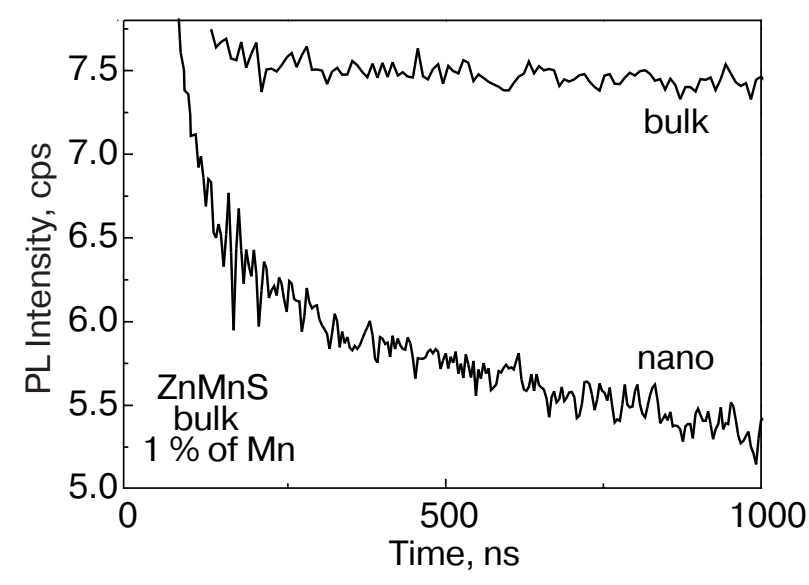

Fig. 3. Fast components of the ${ }^{4} T_{1}$ to ${ }^{6} A_{1}$ PL decay (first $\mu \mathrm{s}$ ) as measured for two $\mathrm{ZnMnS}$ samples (bulk sample and nanocrystals) with $1 \% \mathrm{Mn}$ fraction. The same excitation conditions were used.

nanocrystals. The spectra were collected at low temperature and at the same excitation conditions. Samples with a low Mn fraction of $1 \%$ were selected for these investigations. For samples with larger Mn fractions $\mathrm{Mn}-\mathrm{Mn}$ spin interactions (spin cross-relaxation) become significant relaxing spin selection rules $[10,11,13,14]$ and thus shortening the PL decay time. Then, a multi-component nonexponential decay arises.

Whereas for bulk samples the fast component of the PL decay only weakly contributes to the overall PL kinetics, this component is dominant in the case of nanocrystals and dominates the PL decay. To proceed further, we should first prove that the observed fast PL decay is a property of the $\mathrm{Mn}^{2+}$ intra-shell PL, as was claimed by Bhargava et al. [5-9], and is not due to a spectral overlap of the ${ }^{4} T_{1}$ to ${ }^{6} A_{1}$ PL with some fast decaying host PL emission, as was postulated by Bol and Meijerink [4]. We measured time-resolved PL spectra to solve this contradiction between models proposed by Bhargava et al. [5-9] and Bol and Meijerink [4]. We compared PL spectra collected through the first $1 \mu$ s and $5 \mathrm{~ms}$ of the PL decay. These spectra are shown in Fig. 2, as measured for $\mathrm{ZnMnS}$ bulk samples with $1 \% \mathrm{Mn}$ fraction. We found that for bulk samples and for nanocrystals the fast and slow PL response is due to the ${ }^{4} T_{1}$ to ${ }^{6} A_{1}$ PL emission. Using time-resolved PL we could thus prove that the fast and slow components of the PL decay are a property of the $\mathrm{Mn}^{2+}$ intra-shell transition. We thus confirm results presented in Ref. 4 that fast PL decay is accompanied by a slow decay, but we reject that these two PL decay components are due to two overlapping PL bands.

\subsection{Spin-dependent mechanisms}

The decay time of $\mathrm{Mn}^{2+}$ intra-shell emission depends on the strength of the spin-orbit interaction in the given compound. This time shortens from milliseconds in $\mathrm{ZnS}: \mathrm{Mn}$ (about $1.8 \mathrm{~ms}$ [3] for cubic phase $\mathrm{ZnS}$ ) to microseconds in CdTe:Mn and ZnTe:Mn [2]. This means that relaxation of the spin selection rules is required for the shortening of the PL decay of the ${ }^{4} T_{1}$ to ${ }^{6} A_{1}$ transition of $\mathrm{Mn}^{2+}$ ions. In further investigations we thus searched for possible mechanisms that can relax the spin selection rules for intra-shell transitions, and thus lead to fast PL decay.

Possible spin-dependent interactions that could speed up the $\mathrm{Mn}^{2+}$ intra-shell decay, were reviewed by us recently [14]. Using two magnetic resonance techniques - conventional ESR and ODMR, we confirmed a significant influence of spin cross-relaxation processes on the rate of spin relaxation in II-Mn-VI compounds. In the ODMR we found that two adjacent $\mathrm{Mn}$ ions can flip their spins, which shortens spin relaxation time and thus results in an enhanced rate of $\mathrm{Mn}^{2+}$ intra-shell transitions [10]. Efficient spin flips of nearest-neighbor $\mathrm{Mn}^{2+}$ ions were also used for the description of intra-shell PL in $\mathrm{ZnMnSe}$ and, in particular, to explain the low sensitivity of this emission to magnetic fields up to $6 \mathrm{~T}$ [16]. The spin-lattice relaxation time decreases with increasing concentration of magnetic component [17], which is due to efficient $\mathrm{Mn}-\mathrm{Mn}$ cross-relaxation. This in turn increases the rate of PL decay of spin-forbidden transitions. Moreover, for heavily doped samples the PL decay of the ${ }^{4} T_{1}$ to ${ }^{6} A_{1}$ intra-shell transition is defined by energy migration among Mn ions $[18,19]$. This is why most of the experiments discussed in the present work we performed for samples with relatively low Mn fractions. In such cases we hoped to separate processes related to $\mathrm{Mn}-\mathrm{Mn}$ cross-relaxation from other spin-dependent interactions.

$\mathrm{Mn}^{2+}$ ions can also exchange their spin excitation with components of a donor-acceptor pair (DAP), as we observed in the ODMR investigations of $\mathrm{ZnMnSe}$ bulk samples [10]. This process is however not efficient in most of the samples, and was observed only for samples co-doped with donor and acceptor impurities introduced to activate DAP transitions.

The processes mentioned above mean that fast and slow components of the intra-shell PL decay should always co-exist and should depend on the Mn fraction in the samples studied. Our estimates indicate, however, that the processes described above are not efficient enough to shorten the PL decay by more than two or three orders of magnitude and not by five orders of magnitude, as claimed in the Ref. 5. Moreover, the role of these processes should be reduced in low-dimensional structures (nanocrystals) rather than en- 
hanced. In small nanocrystals a significant part of the $\mathrm{Mn}$ ions is present in near-surface parts of the samples, where they have a reduced number of Mn neighbors. In such a case slower rates of spin relaxation are expected, as we confirmed in our ESR investigations [14].

\subsection{Mn-free carriers spin-flip interactions}

Above we showed that PL decay becomes faster in doped nanocrystals, which is in agreement with the results reported by Bhargava and co-workers [5-9]. We also confirmed the observation of Bol and Meijerink [4] that the fast and slow components of the PL decay co-exist. We rejected however the possibility that the fast PL decay is not the property of the ${ }^{4} T_{1}$ to ${ }^{6} A_{1}$ intra-shell PL, but is due to another underlying PL emission band.

The crucial question arose: how to explain the coexistence of various PL decay components. As already mentioned, Bol and Meijerink claimed that the QCA model is incorrect if a slow ( $\mathrm{ms}$ for $\mathrm{ZnMnS}$ ) PL decay is observed. This statement is correct if the QCA model means quantum confinement of impurity wave functions, as claimed in the version of the model proposed in Ref. 8 for RE ions. However, if the version proposed in [7] is the essence of the QCA model, we can easily explain the co-existence of the fast and slow PL decay components. Let us assume that the fast PL decay is only seen if free carriers are co-excited. Once free carriers decay, are trapped etc., the mechanism of the ${ }^{4} T_{1}$ to ${ }^{6} A_{1}$ PL enhancement is deactivated and the slow PL decay of the $\mathrm{Mn}^{2+}$ intra-shell PL should be observed.

In further research we verified this hypothesis by comparing the decay characteristics of PL emissions related to the presence of free carriers and of the $\mathrm{Mn}^{2+}$ intra-shell emission. These investigations were performed for two systems in which such emissions were observed together - for CdMnTe quantum dots [10] and for CdMnS nanocrystals [15]. For ZnMnS free carriers are trapped at various defect levels and thus DAP emission arises rather than band edge PL. Thus, we could not detect any free carrier related emission (band-to-band, free excitonic or free-to-trapped) to perform similar investigations.

The relevant results are discussed elsewhere $[10,15]$ and thus are not repeated here. We only summarize the conclusions drawn from these investigations. Both for CdMnTe [10] and CdMnS [15] we observed that the fastest component of the PL decay of the $\mathrm{Mn}^{2+}$ intra-shell emission correlates with the decay time of free carriers in the system studied $[10,14,15]$. Once free carriers recombine, the efficiency of the $\mathrm{Mn}^{2+}$ intra-shell PL drops. Then, as a consequence of strong spin-dependent $\mathrm{Mn}$-free-carrier interactions, this drop is a measure of the free-carrier lifetime in the system studied.

\section{Summary and conclusions}

Our extensive investigations led to the surprising conclusion that the fastest component in the PL decay of the $\mathrm{Mn}^{2+}$ intra-shell PL is a measure of lifetime of free carriers (free-carrier-related emissions) in low-dimensional (quantum dots and nanocrystals) systems. This fastest component of the PL decay of the ${ }^{4} T_{1}$ to ${ }^{6} A_{1}$ intra-shell emission is thus not due to «a combination of new quantum physics and novel chemical synthesis» as claimed in Ref. 7, but is a consequence of very efficient $\mathrm{Mn}$ - free-carrier interactions.

The above is only possible if the intra-shell emission is significantly enhanced once free carriers are co-excited. This effect is pronounced in nanocrystals, but is also seen in bulk samples. There are two possible explanations of the PL enhancement caused by interactions of $\mathrm{Mn}$ ions with free carriers:

a) $\mathrm{Mn}$ - free-carrier spin flip or

b) $s p-d$ hybridization.

We favour the former interaction (efficient $\mathrm{Mn}$ - free-carrier spin flip), rather than carrier-related strong $s p-d$ hybridization, which was postulated in the QCA model. High efficiency of such spin-flip interactions was proved experimentally in several systems [20-23]. Moreover, the $s p-d$ hybridization, postulated in the QCA model, may not be so efficient as was assumed originally. This interaction can shorter the PL decay by only two orders in magnitude, as was calculated recently in Ref. 24, i.e., this interaction is of a similar efficiency as other spin-dependent interactions, including $\mathrm{Mn}-\mathrm{Mn}$ cross-relaxation.

Even though we can relate the fastest component of the PL decay to lifetime of free carriers or free carrier-related transitions, rather than «a new quantum mechanics», still the PL enhancement of the ${ }^{4} T_{1}$ to ${ }^{6} A_{1}$ transition is a reality and needs explanation. It has been confirmed experimentally that there is a clear relation between the size of nanocrystals and the PL intensity, as was shown in Ref. 5. A possible reason for the enhanced rate of the ${ }^{4} T_{1}$ to ${ }^{6} A_{1}$ intra-shell PL was proposed in Ref. 7. It was proposed that confinement enhances the rate of host-impurity energy transfer to transition metal or rare earth ions. We checked such a possibility by comparing the PL excitation (PLE) spectra for two samples with $1 \% \mathrm{Mn}$ fraction - for a bulk sample and for nanocrystals of $\mathrm{ZnMnS}$. The relevant results are shown in Fig. 4. Whereas for the bulk sample the PLE spectrum consists of several peaks corresponding to transitions from ${ }^{6} A_{1}\left(\mathrm{Mn}^{2+}\right.$ ground state $)$ to the excited ${ }^{4} G\left({ }^{4} T_{1},{ }^{4} T_{2},{ }^{4} E\right.$, and $\left.{ }^{4} A_{1}\right)$ and ${ }^{2} I\left({ }^{2} T_{2}\right)$ states, as observed previously (see, e.g., [25]), the PLE for nanocrystals is dominated by a host excitation transition. Intra-shell excitation is 


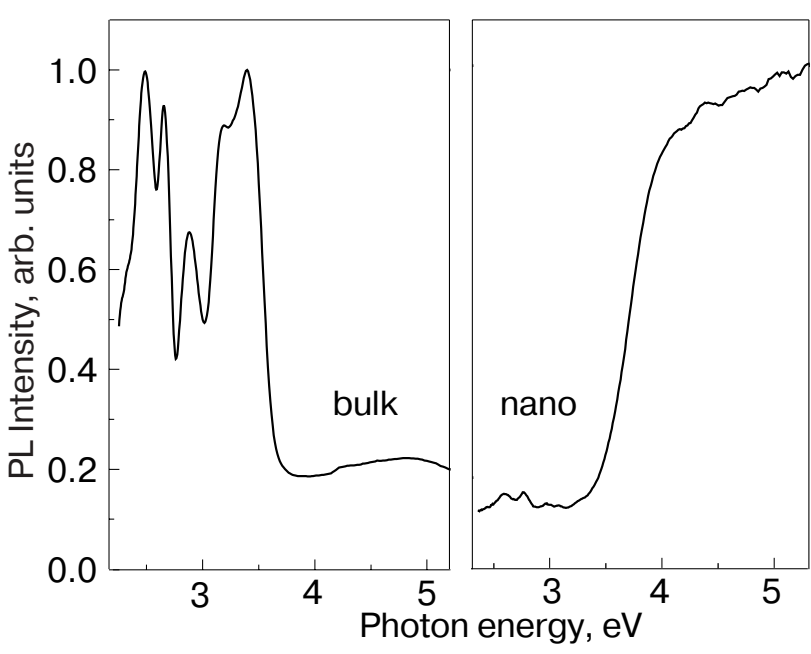

Fig. 4. Room temperature PLE spectra of the ${ }^{4} T_{1}$ to ${ }^{6} A_{1}$ intra-shell emission as measured for two $\mathrm{ZnMnS}$ samples for bulk sample (left) and for nanocrystals (right) with $1 \% \mathrm{Mn}$ fraction. The same excitation conditions were used.

very inefficient as compared to band-to-band excitation (Fig. 4).

This apparent difference between the PLE spectra indicates an enhanced rate of energy pumping to $\mathrm{Mn}^{2+}$ ions in nanocrystals. In consequence, the emission intensity should rise. This enhanced efficiency of host-to- $\mathrm{Mn}^{2+}$ energy pumping and also confinement-enhanced $\mathrm{Mn}$ - free-carrier spin-flip interactions are responsible for increased quantum efficiency of the ${ }^{4} T_{1}$ to ${ }^{6} A_{1}$ intra-shell PL in nanocrystals. Most likely the same processes explain the enhanced rate of the intra-shell PL of RE-doped nanocrystals.

Summarizing, even though we demonstrate that the fast PL decay is not a direct property of the $\mathrm{Mn}^{2+}$ transition, but is a measure of the free-carrier lifetime, we confirm that the ${ }^{4} T_{1}$ to ${ }^{6} A_{1}$ intra-shell PL is enhanced in nanocrystals. We relate this effect to very efficient spin-flip interactions between $\mathrm{Mn}^{2+}$ ions and free carriers and to efficient energy pumping from host to $\mathrm{Mn}^{2+}$ ions. The present results mean that doped nanocrystals are promising materials for various applications, including those as fluorescence labels in biology and medicine.

This work was partly supported by grant No 1 P03B 09030 of MEiN, Poland.

1. E.M. Goldys, K. Drozdowicz-Tomsia, G. Zhu, Hong $\mathrm{Yu}$, Sun Jinjun, Motlan, and M. Godlewski, Optica Applicata (in press).

2. M.V. Artemyev, L.I. Gurinovich, A.P. Stupak, and S.V. Gaponenko, Physica Status Solidi (b) 224, 191 (2001).

3. A. Balzarotti, M. De Crescenzi, R. Messi et al., Phys. Rev. B36, 7428 (1987).
4. A.A. Bol and A. Meijerink, Phys. Rev. B58, R15997 (1998).

5. R.N. Bhargava and D. Gallagher, Phys. Rev. Lett. 72, 416 (1994).

6. T.A. Kennedy, E.R. Glaser, P.B. Klein, and R.N. Bhargava, Phys. Rev. B52, R14356 (1995).

7. R.N. Bhargava, J. Luminescence 70, 85 (1996).

8. R.N. Bhargava, V. Chhabra, B. Kulkarni, and J.V. Veliadis, Physica Status Solidi (b) 210, 621 (1998).

9. R.N. Bhargava, V. Chhabra, T. Som, A. Ekimov, and N. Taskar, Physica Status Solidi (b) 229, 897 (2002).

10. M. Godlewski, V.Yu. Ivanov, A. Khachapuridze, and S. Yatsunenko, Physica Status Solidi (b) 229, 533 (2002).

11. M. Godlewski, S. Yatsunenko, A. Khachapuridze, V.Yu. Ivanov, Z. Golacki, G. Karczewski, P.J. Bergman, P.J. Klar, W. Heimbrodt, and M.R. Phillips, J. Alloys and Compounds 380, 45 (2004).

12. M. Godlewski, S. Yatsunenko, V.Yu. Ivanov, A. Khachapuridze, K. Świątek, E.M. Goldys, M.R. Phillips, P.J. Klar, and W. Heimbrodt, Acta Physica Polonica A107, 65 (2005).

13. M. Godlewski, Optica Applicata (in press).

14. M. Godlewski, S. Yatsunenko, and V.Yu. Ivanov, Izraeli J. Chem. (in press).

15. M. Godlewski, S. Yatsunenko, K. Drozdowicz-Tomsia, E.M. Goldys, M.R. Phillips, P.J. Klar, and W. Heimbrodt, Acta Physica Polonica A108, 681 (2005).

16. J.F. MacKay, W.M. Becker, J. Spalek, and U. Debska, Phys. Rev. B42, 1743 (1990).

17. D. Scalbert, J. Chernogora, and C. Benoit a la Guillaume, Solid State Commun. 66, 571 (1988).

18. E. Muller, W. Gebhardt, and V. Gerhardt, Phys. Status Solidi (b) 113, 209 (1982).

19. V.F. Agekyan, Yu.V. Rud', and R. Schwabe, Sov. Phys. Solid State 29, 970 (1987).

20. M. Godlewski, A. Wittlin, R.R. Galazka, B. Monemar, T. Gregorkiewicz, C.A.J. Ammerlaan, P.H.M. van Loosdrecht, and J.A.A.J. Perenboom, in: The Physics of Semiconductors, M. Scheffler and R. Zimmermann (eds.), Proc. XXIII Intern. Conference ICPS'1996, Berlin (1996), World Scientific, Singapore (1996), Vol. 1, p. 393.

21. S.M. Ryabchenko, Yu.G. Semenov, and O.V. Terletskii, Sov. Phys. JETP 55, 557 (1982).

22. B. König, I.A. Merkulov, D.R. Yakovlev, W. Ossau, S.M. Ryabchenko, M. Kutrowski, T. Wojtowicz, G. Karczewski, and J. Kossut, Phys. Rev. B61, 16870 (2000).

23. S. Yatsunenko, K. Światek, V.Yu. Ivanov, A. Khachapuridze, and M. Godlewski, Physica Status Solidi (c) 2, 1184 (2005).

24. Nguyen Que Huong and J.L. Birman, Phys. Rev. B69, 085321 (2004).

25. W. Giriat, Phys. Status Solidi (b) 136, K129 (1986). 